\title{
Inhibition of Endotoxin-induced Priming of Human Neutrophils by Lipid $X$ and 3-Aza-Lipid $X$
}

\author{
Robert L. Danner, Keith A. Joiner," and Joseph E. Parrillo \\ Critical Care Medicine Department and ${ }^{*}$ National Institute of Allergy and Infectious Diseases, National Institutes of Health, \\ Bethesda, Maryland 20892
}

\begin{abstract}
Lipid X, a precursor of lipid A (the toxic moiety of endotoxin), has been shown to protect animals from the lethal effects of endotoxin challenge. We investigated the mechanism of action of lipid $X$ and 3-aza-lipid $X$, a diamino-analogue, in vitro, using the ability of lipopolysaccharide (LPS) to prime neutrophils for an enhanced release of toxic oxygen radicals. Lipid $X$ and 3-aza-lipid $X$ inhibited LPS-induced neutrophil priming in a concentration-dependent manner. At high concentrations, 3-aza-lipid $X$ was a partial agonist of priming. Lipid $X$ was found to inhibit LPS-induced priming by directly interacting with the neutrophil in contrast to polymyxin $B$, which neutralized LPS by binding to it. Increasing concentrations of lipid $X$ shifted the LPS dose response curve of neutrophils rightward but did not prevent maximum priming at higher LPS concentrations, a finding consistent with competitive inhibition. These results suggest that lipid $X$, a compound structurally related to lipid A, may block neutrophil priming by competing with LPS for cellular binding sites. Lipid $X$ appears to have a novel mechanism of inhibiting LPS effect and may have efficacy in the treatment of gram-negative sepsis.
\end{abstract}

\section{Introduction}

Septic shock caused by gram-negative bacteria has a high mortality despite appropriate antibiotics and modern critical care support (1-4). Endotoxin, a lipopolysaccharide associated with the outer membrane of gram-negative bacteria, has been implicated in the pathogenesis of this often fatal syndrome (5-7). Because of the presumed role of endotoxin in septic shock, investigators have studied therapeutic interventions aimed at alleviating the effects of endotoxin or its secondary mediators. Corticosteroids, naloxone, cyclooxygenase inhibitors, endotoxin inhibitors (polymyxin B), and anti-core endotoxin antibodies have been investigated, but their use and efficacy in human septic shock remain controversial (8-16).

Portions of this work were presented at the American Federation for Clinical Research, Washington, DC, April 1986 (1986. Clin. Res. 34:515A), the American Federation for Clinical Research Eastern Regional Meeting, New York, September 1986 (1986. Clin. Res. 34:877A), and the 26th Interscience Conference on Antimicrobial Agents and Chemotherapy, New Orleans, LA, September 1986 (1986. Abstracts of the 26th ICAAC. 492).

Address correspondence to Robert L. Danner, M.D., Critical Care Medicine Department, Clinical Center, National Institutes of Health, Building 10, Room 10D48, Bethesda, MD 20892.

Received for publication 11 December 1986 and in revised form 15 April 1987.

The Journal of Clinical Investigation, Inc.

Volume 80, September 1987, 605-612
Recently, novel glucosamine-derived phospholipids related to lipid $\mathrm{A}$, the toxic moiety of endotoxin, have been identified from mutant Escherichia coli (17-19) and chemically synthesized $(20,21)$. Lipid X (2,3 diacyl glucosamine 1-phosphate), the prototype, is a monosaccharide precursor in the biosynthetic pathway of lipid A $(22,23)$. Lipid X has some of the immunomodulating properties of endotoxin (24-26) but is much less toxic $(18,27)$. This compound has been shown to protect mice and sheep from the lethality of endotoxin administration, though the mechanism of this protective effect is unknown (27-29). The structural similarity between lipid $X$ and lipid A suggests that lipid $X$ might be mediating its protective effect by specifically blocking endotoxin-triggered events, and possibly even competing directly with LPS $^{1}$ for cellular binding sites, a hypothesis that can be tested in an in vitro system.

LPS has been reported to "prime" neutrophils, resulting in a markedly enhanced respiratory burst when stimulated with a variety of activators (30). In the present study, lipid $X$ and its diamino analog, 3-aza-lipid $X$, were evaluated for their ability either to prime or to prevent LPS-mediated priming of human neutrophils. We report here that lipid $X$ can prevent neutrophil priming by LPS in a concentration-dependent manner and that 3-aza-lipid $X$ appears to be a partial agonist of neutrophil priming. Furthermore, lipid $X$ shifts the LPS dose response curve of neutrophils to the right, suggesting that lipid $X$ may be a competitive inhibitor of LPS-induced priming. This class of compounds appears to have a unique anti-endotoxin effect which may have therapeutic efficacy in the treatment of septic shock and offers a novel approach to the management of this highly lethal disorder.

\section{Methods}

Endotoxin, lipid $X$ and 3-aza-lipid $X$. Highly purified protein-free LPS extracted from the Salmonella minnesota Re595 mutant and Salmonella typhimurium (List Biological Laboratories, Campbell, CA) was prepared as a stock solution $(1 \mathrm{mg} / \mathrm{ml})$ in sterile pyrogen-free water sonicated for 4 min at maximum output (model 16-850, Virtis Co., Gardiner, NY), and then stored in small aliquots at $-70^{\circ} \mathrm{C}$. On the day of use the LPS was thawed, sonicated for an additional $2 \mathrm{~min}$, and adjusted to the desired final concentration in Hanks' balanced salt solution (HBSS; Gibco, Grand Island, New York) without calcium and magnesium ( - ).

Lipid X (2,3 diacyl glucosamine 1-phosphate) and 3-aza-lipid X (2,3 diacyl diaminoglucose 1-phosphate) were supplied as crystaline di-Tris salts by Sandoz Forschungsinstitut, Vienna, Austria (Fig. 1). These compounds were dissolved, sonicated, stored, and prepared for use in an identical fashion to the LPS.

1. Abbreviations used in this paper: FMLP, $N$-formyl-methionyl-leucyl-phenylalanine; $k_{1 / 2}$, concentration of LPS in $\mathrm{ng} / \mathrm{ml}$ resulting in a half-maximal priming effect; LPS, lipopolysaccharide. 


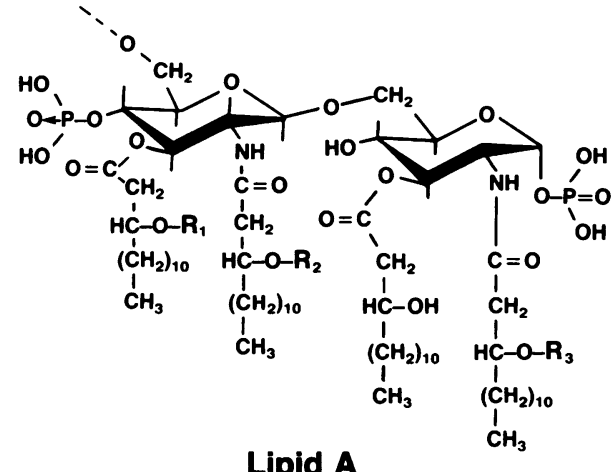

Lipid A

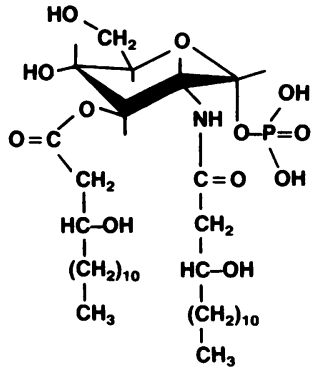

Lipid X

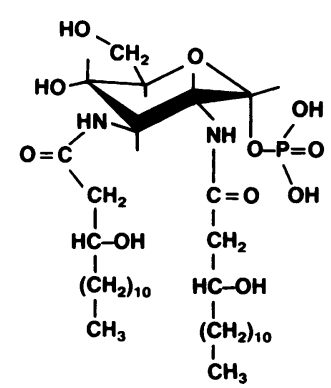

3-Aza-Lipid $X$
Figure 1. Chemical structure of lipid A, lipid X, and 3-aza-lipid X. Lipid $\mathrm{X}$ is a monosaccharide precursor of lipid $\mathrm{A}$, and 3-aza-lipid $\mathrm{X}$ is a diamino-analog of lipid $X . R_{1}$ is usually a lauroyl and $R_{2}$ a myristoyl moiety. $R_{3}$ can be either a hydrogen or palmitoyl group, depending on the source of the lipid A.

Reagents. All reagents, buffers, and plasticware used through the priming step were determined to be pyrogen-free by a chromogenic limulus lysate assay (Whittaker M. A. Bioproducts, Walkersville, MD) sensitive to $10 \mathrm{pg} / \mathrm{ml}$ of United States Standard Endotoxin. This included testing of Ficoll-Hypaque previously reported to be contaminated with LPS (31). The Ficoll-Hypaque used in these experiments contained $<10 \mathrm{pg} / \mathrm{ml}$ of LPS. Laboratory glassware and sonicator microtips were thoroughly cleaned and then baked in dry heat for $4 \mathrm{~h}$ at $180^{\circ} \mathrm{C}$ to render them free of contaminating LPS (32).

Neutrophil isolation. Human neutrophils were isolated from the venous blood of normal volunteers using standard techniques. The blood was anticoagulated with $5 \mathrm{U} / \mathrm{ml}$ of sterile pyrogen-free, preservative-free heparin (porcine intestine; Weddel Pharmaceuticals, Wrexham, UK). Pyrogen-free dextran (United States Biochemical Corporation, Cleveland, $\mathrm{OH}$ ) of $200,000-300,000 \mathrm{~mol}$ wt was prepared as a $3.0 \%$ solution in HBSS(-). This was added to the blood in a 1:2 ratio and the blood-dextran mixture was allowed to stand at a slight angle for $20 \mathrm{~min}$. The straw-colored supernatant was then collected and layered onto a half volume of Ficoll-Hypaque (Lymphocyte Separation Media; Litton Bionetics Inc., Charleston, SC). This was then centrifuged at $250 \mathrm{~g}$ (model CRU-5000, International Equipment Co., Needham Hgts, MA) for $30 \mathrm{~min}$ at $18^{\circ} \mathrm{C}$. The upper layers were discarded and the pellet was subjected to cold, sterile, pyrogen-free water $(4 \mathrm{ml})$ for $30 \mathrm{~s}$ to lyse residual red blood cells followed by addition of hypertonic $3 \%$ saline $(2 \mathrm{ml})$. This solution was then diluted with cold, sterile HBSS(-) and centrifuged at $250 \mathrm{~g}$ for $10 \mathrm{~min}$ at $4^{\circ} \mathrm{C}$. The pellet was retained, suspended in cold HBSS(-), and the cell concentration was adjusted to $1.0 \times 10^{7} / \mathrm{ml}$ by cell quantitation in a counter (Coulter Electronics Inc., Hialeah, FL). Neutrophils isolated by this method were $>98 \%$ pure by Wrights stain and $>98 \%$ viable by trypan blue exclusion.

Neutrophil priming by LPS. Neutrophils suspended in HBSS(-) were mixed 1:1 with additional buffer with or without LPS to achieve a final concentration of $5 \times 10^{6} \mathrm{cells} / \mathrm{ml}$ and incubated in a shaking water bath at $37^{\circ} \mathrm{C}$ according to the method of Guthrie (30). The optimal incubation time for priming was $60 \mathrm{~min}$, confirming the findings previously reported (30). After incubation, cells were examined microscopically to ascertain that clumping did not occur. Trypan blue exclusion always showed the neutrophils to be $>92 \%$ viable after incubation.

Superoxide $\left(\mathrm{O}_{2}^{-}\right)$assay. Superoxide production was determined by a modification of previously described methods utilizing superoxide dismutase (bovine; Sigma Chemical Co., St. Louis, MO)-inhibitable reduction of cytochrome $c$ (type VI; Sigma Chemical Co.) $(33,34)$. A $200-\mu \mathrm{l}$ aliquot of the incubation mixture (1.0 $\times 10^{6}$ neutrophils), $50 \mu \mathrm{l}$ of stock cytochrome $c(1.0-\mathrm{mM}$ solution in HBSS with calcium and magnesium ( + ); Gibco), and $700 \mu \mathrm{l}$ of $\mathrm{HBSS}(+)$ were combined in a plastic test tube and gently mixed. $N$-formyl-methionyl-leucyl-phenylalanine (FMLP; Sigma Chemical Co.) was stored at $-70^{\circ} \mathrm{C}$ in HBSS(+) and $10 \%$ dimethylsulfoxide (Sigma Chemical Co.) at a concentration of $10^{-4} \mathrm{M}$. On the day of use, the FMLP stock solution was diluted 1:5 with $\mathrm{HBSS}(+)$, and $50 \mu \mathrm{l}$ were placed into a clean 1-cc glass cuvette (Gilford Instrument Laboratories, Inc., Oberlin, $\mathrm{OH} ; 1,245$ $\times 105$ ) maintained at $37^{\circ} \mathrm{C}$ in a spectrophotometer (Gilford Instrument Laboratories, Inc.; model 2600). The cell-cytochrome $c$ mixture was then rapidly pipetted into the cuvettes and the absorption at $\mathbf{5 5 0}$ $\mathrm{nm}$ was adjusted to zero. Changes in absorption were continuously recorded on a strip chart recorder (Gilford Instrument Laboratories, Inc.; model 6051).

The peak absorption change and the absorption change per minute were converted to nanomoles of cytochrome $c$ reduced, using the extinction coefficient of $2.1 \times 10^{4} \mathrm{M}^{-1} \mathrm{CM}^{-1}$ previously reported (35) and expressed as nanomoles $\mathrm{O}_{2}^{-} / 10^{6}$ neutrophils (total production) and nanomoles $\mathrm{O}_{2}^{-} / 10^{6}$ neutrophils per minute (rate of production), respectively. Some specimens were stimulated in the presence of superoxide dismutase $10 \mu \mathrm{g} / \mathrm{ml}$, to ascertain that the absorption changes measured were due specifically to $\mathrm{O}_{2}^{-}$release. The activity of the Salmonella typhimurium LPS (smooth parent strain) was confirmed in the priming assay, but the Salmonella minnesota Re595 LPS (core endotoxin) was used in the subsequent experiments reported here.

Effect of lipid $X$, 3-aza-lipid $X$, and polymyxin $B$ on neutrophil priming. Lipid $X$ and 3-aza-lipid $X$ were added to the cell suspension at room temperature $15 \mathrm{~min}$ before initiation of incubation with LPS unless specified otherwise. Polymyxin B (Upjohn Co., Kalamazoo, MI) was reconstituted from powder to the desired concentration in HBSS(-) on the day of use. Neutrophils incubated with lipid X, 3-aza-lipid $\mathrm{X}$, or polymyxin $\mathrm{B}$ did not differ in $\mathrm{O}_{2}^{-}$release from cells incubated in buffer alone upon stimulation with either FMLP or phorbol-myristate-acetate (PMA; Sigma Chemical Co.; 500 ng/ml).

Statistics. Means are expressed plus or minus the standard error of the mean (SEM) in the text and figures where applicable. Differences between experimental groups were analyzed using paired and unpaired Student $t$ tests where indicated. Data trends were analyzed with the Mann test for trend (36) or the Bartholomew test (37) as stated.

\section{Results}

We initially confirmed previous observations showing that LPS enhances the $\mathrm{O}_{2}^{-}$production of neutrophils exposed to FMLP (30). In 10 paired experiments, neutrophils from different donors incubated with LPS $(10 \mathrm{ng} / \mathrm{ml})$ for $60 \mathrm{~min}$ followed by activation with FMLP released $18.16 \pm 2.57 \mathrm{nmol}$ $\mathrm{O}_{2}^{-} / 10^{6}$ neutrophils compared with $3.20 \pm 0.63 \mathrm{nmol} \mathrm{O}_{2}^{-} / 10^{6}$ neutrophils when cells were incubated in pyrogen-free buffer alone (mean \pm SEM; $P<0.005$ ). Also, as previously reported, LPS priming of neutrophils was noted to augment both total $\mathrm{O}_{2}^{-}$production and rate of $\mathrm{O}_{2}^{-}$production by stimulated cells. Except where indicated, the data reported here are expressed as the total amount of $\mathrm{O}_{2}^{-}$released. 


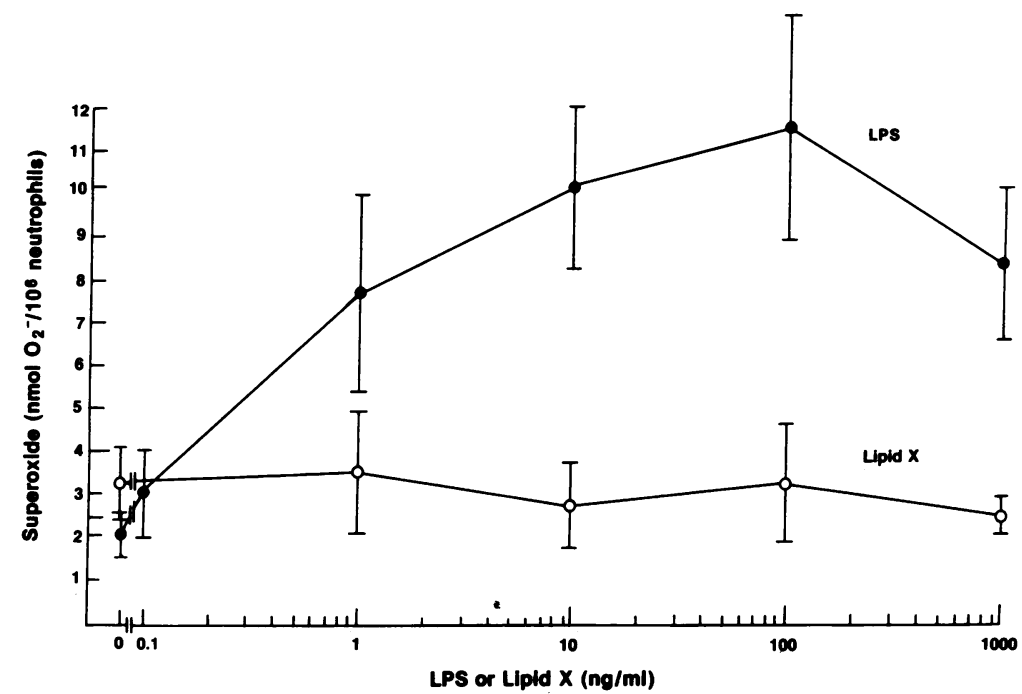

Figure 2. Release of $\mathrm{O}_{2}^{-}$by neutrophils after incubation with increasing concentrations of LPS ( $\bullet$ ) or lipid X (0). The neutrophils were incubated at $37^{\circ} \mathrm{C}$ for $60 \mathrm{~min}$ followed by simulation with FMLP $\left(10^{-6} \mathrm{M}\right)$. The results represent mean \pm SEM. The maximal response was determined for each preparation of neutrophils. Mean maximal responses for the two treatment groups were compared using an unpaired Student's $t$ test $(P<0.01)$.
The generation of $\mathrm{O}_{2}^{-}$by neutrophils was dependent on the concentration of LPS (Fig. 2). LPS at $1 \mu \mathrm{g} / \mathrm{ml}$ caused a small but significant (paired Student's $t$ test; $P<0.05$ ) decrease in $\mathrm{O}_{2}^{-}$ production from the peak level and an insignificant fall in neutrophil viability, as determined by trypan blue exclusion. Lipid $\mathrm{X}$, in contrast, had no effect on FMLP-stimulated $\mathrm{O}_{2}^{-}$ release from neutrophils, even at concentrations as high as 1 $\mu \mathrm{g} / \mathrm{ml}$ (Fig. 2). Neutrophils incubated with this concentration of lipid X were identical to control cells exposed only to pyrogen-free buffer in respect to clumping, viability, and PMA stimulated $\mathrm{O}_{2}^{-}$release. Lipid $\mathrm{X}$ could not be evaluated at levels $>2 \mu \mathrm{g} / \mathrm{ml}$ because of clumping of cells at these higher concentrations. This clumping was considered nonspecific because addition of lipid $X(>2 \mu \mathrm{g} / \mathrm{ml})$ to neutrophils in an aggregometer at $37^{\circ} \mathrm{C}$ resulted in a slow increase in light transmittance over $60 \mathrm{~min}$ and not a typical stimulated aggregation response as characteristically seen with a variety of activators such as PMA or C5a. Control neutrophils and those exposed to lower lipid $\mathrm{X}$ concentrations $(<2 \mu \mathrm{g} / \mathrm{ml})$ did not show clumping by microscopic examination or aggregometry.

In separate paired experiments, lipid $\mathrm{X}$, at a concentration achievable in vivo (27) was tested in the priming assay. Again, lipid $\mathrm{X}$ alone $(1 \mu \mathrm{g} / \mathrm{ml})$ had no effect on the neutrophil respiratory burst, but cells incubated with LPS $(10 \mathrm{ng} / \mathrm{ml})$ produced significantly $(P<0.005)$ more $\mathrm{O}_{2}^{-}$than either the buffer or lipid X exposed cells (Table I). In contrast, incubation of neu-

Table I. Effect of Lipid X on Neutrophil Priming with or without LPS

\begin{tabular}{lllll}
\hline \multirow{2}{*}{$\begin{array}{l}\text { No. of } \\
\text { experiments }\end{array}$} & \multicolumn{4}{l}{ Superoxide (nmol $\mathrm{O}_{2}^{-} / 10^{6}$ neutrophils) } \\
\cline { 2 - 5 } & Buffer & Lipid X & LPS & LPS and lipid X \\
\hline 7 & $3.35 \pm 0.45$ & $3.16 \pm 0.40^{*}$ & $14.67 \pm 2.50$ & - \\
6 & $3.59 \pm 0.39$ & - & $16.76 \pm 2.41$ & $6.30 \pm 1.11^{\ddagger}$
\end{tabular}

Neutrophils incubated for $60 \mathrm{~min}$ in buffer alone or LPS $(10 \mathrm{ng} / \mathrm{ml})$, and/or lipid $X(1 \mu \mathrm{g} / \mathrm{ml})$, followed by FMLP $\left(10^{-6} \mathrm{M}\right)$ activation.

* Cells incubated in buffer or lipid $\mathrm{X}$ released similar amounts of $\mathrm{O}_{2}^{-}$ $(P=\mathrm{NS})$, but less than neutrophils exposed to LPS alone $(P<0.005)$. ${ }^{\ddagger}$ Neutrophils incubated in the pesence of lipid $X$ and LPS produced less $\mathrm{O}_{2}^{-}$than those incubated with LPS alone $(P<0.005)$. trophils with both lipid $X(1 \mu \mathrm{g} / \mathrm{ml})$ and LPS $(10 \mathrm{ng} / \mathrm{ml})$ resulted in a profound $(P<0.005)$ inhibition of $\mathrm{O}_{2}^{-}$release when compared with cells treated with LPS alone (Table I).

Next, the concentration dependence of this blocking effect of lipid X on LPS-induced neutrophil priming was examined. Lipid $X$ in concentrations from $100 \mathrm{ng} / \mathrm{ml}$ to $2 \mu \mathrm{g} / \mathrm{ml}$ was added to neutrophils $15 \mathrm{~min}$ before the start of incubation with LPS $(10 \mathrm{ng} / \mathrm{ml})$. As can be seen in Fig. 3, lipid X prevented LPS from priming neutrophils in a concentration-dependent manner with nearly complete inhibition at $2 \mu \mathrm{g} / \mathrm{ml}$.

Fig. 4 shows the temporal relationship between the addition of lipid X and its ability to inhibit LPS-induced neutrophil priming. Again, lipid X $(1 \mu \mathrm{g} / \mathrm{ml})$ significantly prevented the enhanced FMLP-stimulated $\mathrm{O}_{2}^{-}$release seen with cells exposed to LPS alone when added $15 \mathrm{~min}$ before the start of incubation. As lipid X was added later and later (after neutrophil exposure to LPS), its ability to prevent priming was diminished, but significant $(P<0.05)$ inhibition of priming was

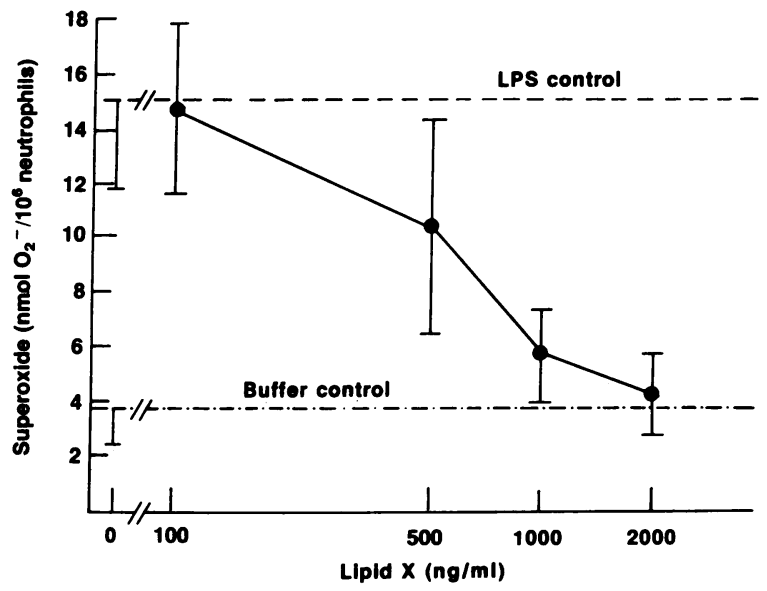

Figure 3. The effect of increasing concentrations of lipid X on LPSinduced neutrophil priming. Neutrophils were pre-exposed to lipid $X$ for $15 \mathrm{~min}$ before incubation with LPS $(10 \mathrm{ng} / \mathrm{ml})$ for $60 \mathrm{~min}$ at $37^{\circ} \mathrm{C}$, followed by activation with FMLP $\left(10^{-6} \mathrm{M}\right)$. (---) $\mathrm{O}_{2}^{-}$produced by LPS-primed neutrophils in the absence of lipid X. (-.---) $\mathrm{O}_{2}^{-}$production by neutrophils incubated in pyrogen-free buffer without LPS or lipid X. Results are mean \pm SEM of three experiments. 


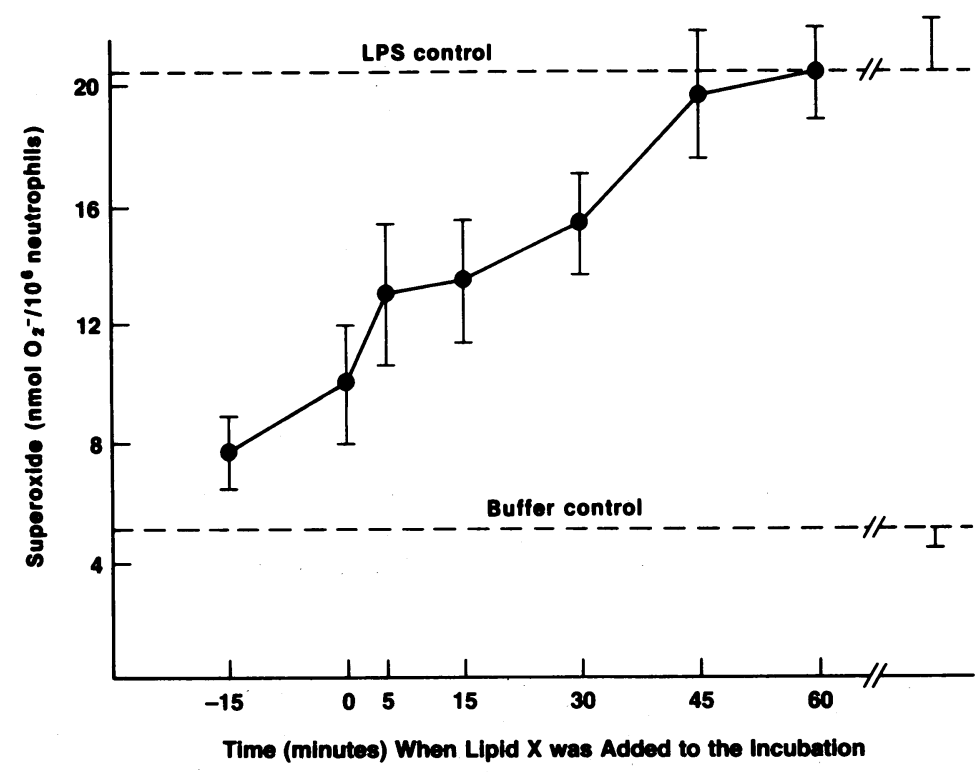

Figure 4. Temporal relationship between the addition of lipid $X$ and its ability to prevent LPS-induced neutrophil priming. Neutrophils were incubated for $60 \mathrm{~min}$ at $37^{\circ} \mathrm{C}$ with LPS $(10 \mathrm{ng} / \mathrm{ml})$. Lipid X $(1 \mu \mathrm{g} / \mathrm{ml})$ was added to the neutrophil-LPS incubation at the time points indicated along the abscissa and $\mathrm{O}_{2}^{-}$production was determined. Dashed lines are as described for Fig. 3. Lipid $\mathrm{X}$ added as late as 30 min into the incubation with LPS significantly $(P$ $<0.05$ ) inhibited priming. still evident when lipid $X$ was added as late as 30 min after the start of incubation with LPS. This contrasts with polymyxin B, which in separate experiments could not prevent neutrophils from becoming fully primed if it was added later than $15 \mathrm{~min}$ into the incubation with LPS (data not shown).

Paired experiments comparing 3-aza-lipid X with lipid X revealed a similar concentration-dependent inhibition of LPSinduced neutrophil priming, though 3-aza-lipid X was slightly less potent on a weight basis (data not shown). Unlike lipid X, 3-aza-lipid $X$ did not cause neutrophil clumping at concentrations up to $10 \mu \mathrm{g} / \mathrm{ml}$. Further studies at these high concentrations showed 3-aza-lipid $X$ to have an apparent priming effect of its own (Fig. 5). In these experiments, 3-aza-lipid $X$ augmented $\mathrm{O}_{2}^{-}$release by neutrophils at concentrations $>2 \mu \mathrm{g} / \mathrm{ml}$ with and without the presence of LPS. At lower concentrations, 3-aza-lipid X significantly inhibited LPS-induced priming of neutrophils, but this was only a partial effect.

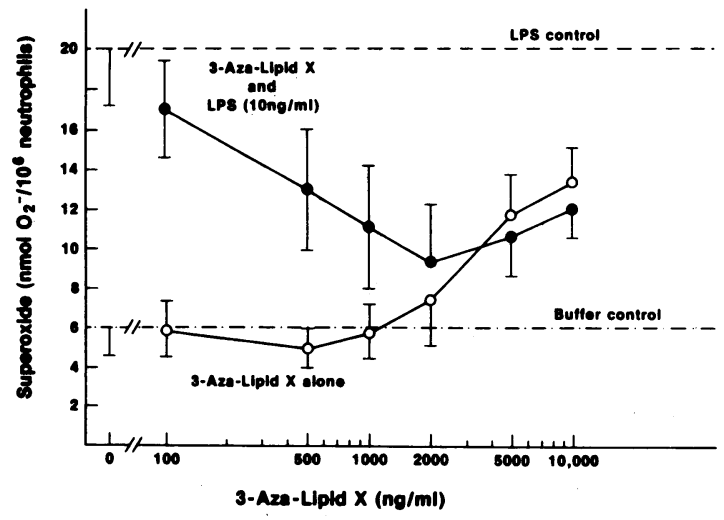

Figure 5. Superoxide production by neutrophils incubated with increasing concentrations of 3-aza-lipid X with $(\bullet)$ and without $(0)$ LPS $(10 \mathrm{ng} / \mathrm{ml})$. FMLP $\left(10^{-6} \mathrm{M}\right)$ was used to stimulate the neutrophils after incubation for $60 \mathrm{~min}$ at $37^{\circ} \mathrm{C}$. Dashed lines are as described for Fig. 3. The difference in response between 3-aza-lipid $X$ alone and in combination with LPS decreased significantly ( $P$ $<0.01$; Mann test for trend) in five of the six subjects.
To further evaluate the mechanism of inhibition of priming by these compounds, the activity of lipid X was compared with that of polymyxin B, a well-characterized inhibitor of endotoxin known to neutralize LPS by binding tightly to it (38). In these experiments (Fig. $6 A$ ) neutrophils were first exposed to either lipid X $(2 \mu \mathrm{g} / \mathrm{ml})$ or polymyxin $B(1 \mu \mathrm{g} / \mathrm{ml})$ for $30 \mathrm{~min}$ at room temperature. Then the cells were centrifuged, washed with pyrogen-free buffer, and incubated at $37^{\circ} \mathrm{C}$ for $60 \mathrm{~min}$ with LPS. Under these conditions, lipid X com-

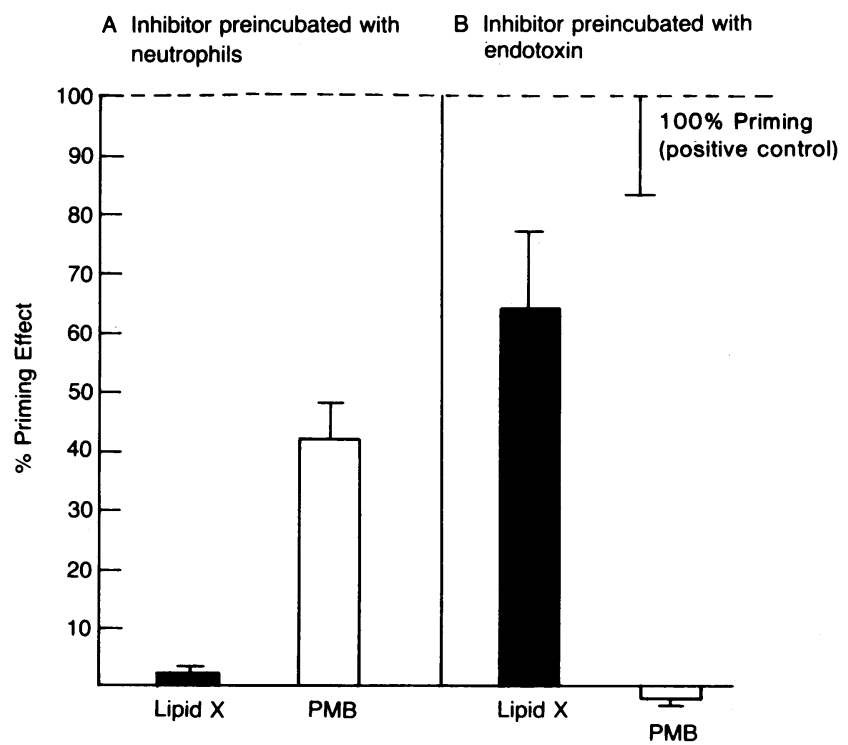

Figure 6. Comparison of the inhibition of LPS $(10 \mathrm{ng} / \mathrm{ml})$-induced neutrophil priming by lipid $X(2 \mu \mathrm{g} / \mathrm{ml})$ and polymyxin $B(1 \mu \mathrm{g} / \mathrm{ml})$. Percent priming effect was calculated from the formula $\left(1-\left[C_{\mathrm{E}}\right.\right.$ $\left.\left.-X / C_{\mathrm{E}}-C_{\mathrm{B}}\right]\right) \times 100$, where $C_{\mathrm{E}}$ is the $\mathrm{O}_{2}^{-}$produced by fully primed neutrophils incubated with LPS alone, $C_{\mathrm{B}}$ is the $\mathrm{O}_{2}^{-}$produced by unprimed neutrophils incubated in pyrogen-free buffer, and $X$ is the $\mathrm{O}_{2}^{-}$ produced by neutrophils incubated with LPS and inhibitor (polymyxin B or lipid X). Results represent mean \pm SEM of nine paired experiments. 


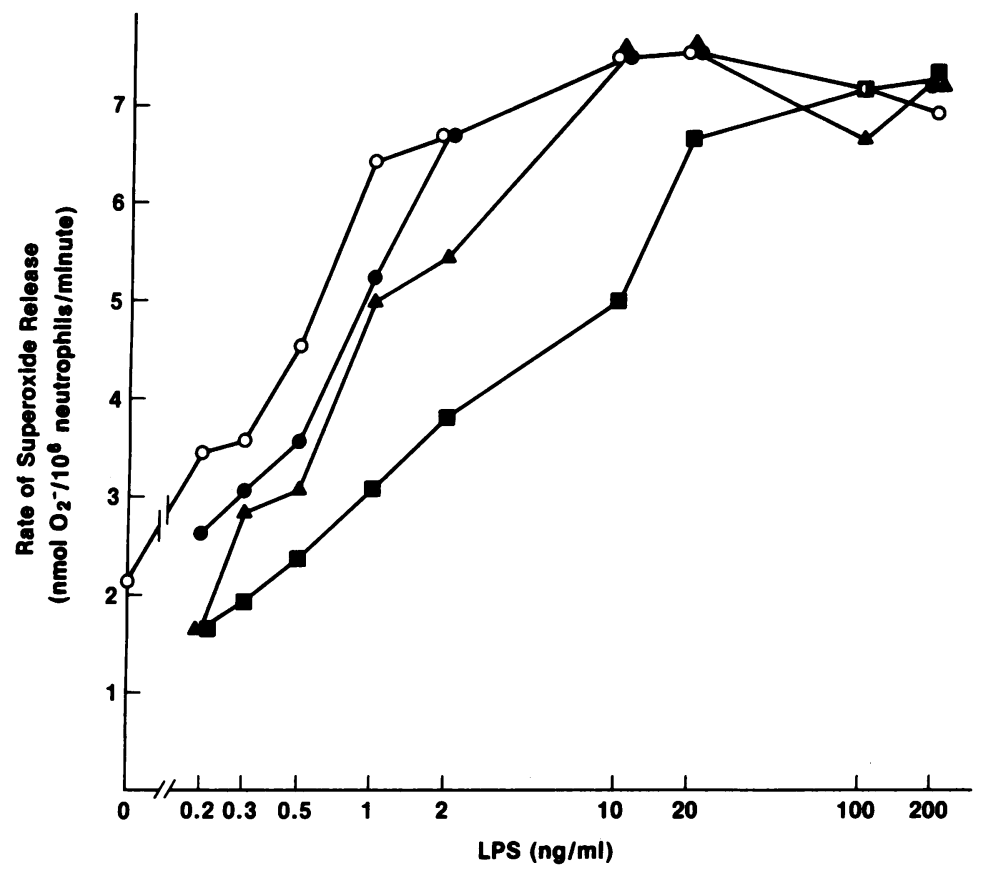

Figure 7. Effect of increasing concentrations of lipid $\mathrm{X}$ on the LPS dose response curve. Serial endotoxin dose response curves were done with $0 \mathrm{ng} / \mathrm{ml}(0), 200 \mathrm{ng} / \mathrm{ml}(\bullet), 400$ $\mathrm{ng} / \mathrm{ml}(\triangle)$, and $800 \mathrm{ng} / \mathrm{ml}(\square)$ of lipid X, respectively. Data are expressed as the initial rate of $\mathrm{O}_{2}^{-}$production from one representative experiment. pletely blocked the priming effect, even though the neutrophils were washed and resuspended in buffer containing only LPS. In contrast, the polymyxin B-treated neutrophils, processed in an identical fashion, still demonstrated significant priming $(42 \%)$.

Next, the possibility that these inhibitors might be interacting directly with LPS to exert their anti-priming effect was investigated. Lipid X or polymyxin B (Fig. 6 B) were combined with LPS for $30 \mathrm{~min}$ before both were added to the neutrophil incubation. Polymyxin B, known to bind tightly to LPS, completely prevented priming, whereas lipid X only partially inhibited priming when allowed to interact directly with LPS before neutrophil incubation. Therefore it appeared that in contrast to polymyxin B, the blocking of LPS-induced priming by lipid $\mathrm{X}$ was primarily due to a direct interaction with the neutrophil and not LPS.

Because many LPS interactions with cell membranes are nonspecific, of high affinity, and neither saturable nor competitive (39), it was necessary to better clarify the nature of the inhibitory effect of lipid X on LPS-induced neutrophil priming. To test the reversibility and specificity of this blocking effect, experiments were conducted to see if higher concentrations of LPS could overcome the inhibitory action of lipid X over a wide dose range. Serial LPS dose response curves were done in the presence of increasing concentrations of lipid X $(0$, 200,400 , and $800 \mathrm{ng} / \mathrm{ml}$ ) and the data expressed as the initial rate of $\mathrm{O}_{2}^{-}$production (nanomoles $\mathrm{O}_{2}^{-} / 10^{6}$ neutrophils per minute). Lipid X shifted the LPS dose response curve to the right, but the maximal priming effect could still be achieved by higher concentrations of LPS (Fig. 7, representative experiment). The concentration of LPS $(\mathrm{ng} / \mathrm{ml})$ required to produce a half-maximal priming response $\left(k_{1 / 2}\right)$ was calculated for each of the lipid X doses in three experiments (Table II). The $k_{1 / 2}$ for LPS-induced neutrophil priming progressively increased with increasing concentrations of Lipid X: $0.62 \pm 0.10,1.03 \pm 0.11$, $1.23 \pm 0.08$, and $3.94 \pm 0.61$, respectively (mean \pm SEM; $P$ $<0.001)$.

\section{Discussion}

Lipid X, a monosaccharide precursor of lipid A (17-19), has been shown to protect animals from the lethality of LPS (27-29) but the mechanism of this protective action is unknown. The structural similarity between lipid X and lipid A, the toxic component of LPS, suggested that the therapeutic action of lipid X might involve the direct inhibition of LPStriggered cellular effects. In this investigation, we have used neutrophil priming by LPS, a recently described functional

Table II. Concentration of LPS Required to Produce a HalfMaximal Priming Effect $\left(k_{1 / 2}\right)$ with Increasing Lipid X

\begin{tabular}{cllll}
\hline & $\mathrm{k}_{1 / 2}$ of LPS & & & \\
\cline { 2 - 5 } Lipid X & Experiment 1 & Experiment 2 & Experiment 3 & Mean \pm SEM $^{*}$ \\
\hline$n g / m l$ & $(n g / m l)$ & $(n g / m l)$ & $(n g / m l)$ & $(n g / m l)$ \\
0 & 0.81 & 0.54 & 0.51 & $0.62 \pm 0.10$ \\
200 & 1.24 & 0.97 & 0.88 & $1.03 \pm 0.11$ \\
400 & 1.34 & 1.08 & 1.28 & $1.23 \pm 0.08$ \\
800 & 4.89 & 4.15 & 2.78 & $3.94 \pm 0.61$
\end{tabular}

$\mathbf{k}_{1 / 2}$ is the concentration of LPS $(\mathrm{ng} / \mathrm{ml})$ producing a half-maximal priming response. The $k_{1 / 2}$ was estimated as follows: (a) Mean rates of $\mathrm{O}_{2}^{-}$release for three experiments were transformed according to the formula: logit $\left(\mathrm{rO}_{2}^{-}\right)=\log _{\mathrm{e}}\left[\left(\mathrm{rO}_{2}^{-}-\mathrm{rO}_{2}^{-} \mathrm{min}\right) /\left(\mathrm{rO}_{2}^{-} \max -\mathrm{rO}_{2}^{-}\right)\right]$, where $\mathrm{rO}_{2}^{-}$min and $\mathrm{rO}_{2}^{-}$max are the minimum and maximum observed rates of $\mathrm{O}_{2}^{-}$release ( $\mathrm{nmol} \mathrm{O}_{2}^{-} / 10^{6}$ neutrophils per $\mathrm{min}$ ) for the experiment. (b) The logit $\left(\mathrm{rO}_{2}^{-}\right)$values were assumed to be linearly related to the corresponding $\log _{10}$ concentrations of LPS and were fitted by least squares. $(c) \log _{10}$ concentrations of LPS required to evoke a half-maximal response were taken to be the negative of the ratio of the intercept and the slope of the fitted line.

* Mean results were tested for upward trend with respect to increasing lipid $X(P<0.001 ;$ Bartholomew test $)$. 
capability of LPS, to study the nature of lipid X-LPS interactions on a cellular level. The data reported here show that lipid $X$ and 3-aza-lipid $X$ are inhibitors of LPS-induced neutrophil priming. The concentrations of lipid $X$ that produce this blocking effect are similar to those required in vivo to prevent mortality in sheep challenged with endotoxin (27).

The events leading to neutrophil priming by LPS have not been fully elucidated, but this action of LPS occurs at concentrations at least one log lower than other reported effects of LPS on neutrophils (40) and in the range of endotoxin concentrations found in blood of patients with septic shock (41, 42). Priming is evident with $10^{-12} \mathrm{M}$ concentrations of LPS, suggestive of a high-affinity interaction. Other direct cellular effects of LPS seen in this dose range include the stimulation of monocytes to produce both cachectin (43) and interleukin 1 (44), endogenous molecules known to be important secondary mediators of the in vivo actions of endotoxin $(45,46)$. Neutrophil activation during sepsis has been linked to tissue injury and the development of multiorgan failure, one of the major causes of death in human septic shock (47). The ability of LPS to prime neutrophils for an enhanced respiratory burst may represent an in vitro correlate of this toxic component of sepsis $(30,48)$.

Work by Guthrie et al. has shown that priming is not blocked by cyclohexamide, an inhibitor of protein synthesis, and does not appear to be due to a change in the number or affinity of FMLP binding sites when determined at $4^{\circ} \mathrm{C}(30)$. Further, priming of neutrophils by LPS was temperature-dependent and appeared to proceed in at least two steps. Neutrophils exposed briefly to LPS at $37^{\circ} \mathrm{C}$, washed, and resuspended in fresh pyrogen-free buffer could still become primed with subsequient incubation at $37^{\circ}$ even though the LPS concentration was reduced below that required for priming. This suggests that attachment of LPS to the cell membrane is rapid, but a subsequent time- and temperature-dependent event is then required for priming to occur. These conditions are highly reminescent of work examining LPS binding to rabbit erythrocytes and platelets (49-51). In those experiments, LPS attached to cell membranes very rapidly, even at $4^{\circ} \mathrm{C}$, followed by insertion of the LPS molecule into the lipid bilayer, a timeand temperature-dependent step (30-60 min at $\left.37^{\circ} \mathrm{C}\right)$. Recently, it has been shown that neutrophils contain acyloxyacyl hydrolases that can selectively remove nonhydroxylated acyl chains from lipid A (52). As the intracellular handling of LPS becomes better understood, it may be found that the metabolism of LPS and related compounds plays a role in either priming or inhibition of priming.

LPS has also been shown to cause the myristoylation of specific macrophage proteins over a brief time period of 20-90 min, and it has been suggested that myristoylation of proteins may represent an early event in both signal transduction and the priming of macrophages by LPS for an enhanced stimulated release of 20:4 arachidonic acid metabolites (53). The time course of protein myristoylation and the similarities between LPS-induced neutrophil and macrophage priming suggest that these processes may be related. Although the mechanism of LPS-induced neutrophil priming is unknown, the current data does not eliminate the possibility that LPS is directly affecting the phosphatidyl-inositol-transmembrane signal pathway (signal transduction) involved in generating the respiratory burst via FMLP (30). If correct, this may have broad implications because many diverse cell types utilize this signal pathway $(54,55)$.

The inhibition of LPS-induced neutrophil priming by lipid X, a compound that is structurally very similar to half of the lipid A molecule, supports the concept that lipid X may be directly antagonizing the action of LPS on neutrophils by interacting with the same cellular site(s) mediating this effect. Lipid X was shown to exert its major anti-LPS effect by interacting directly with the neutrophil, unlike polymyxin B which appears to produce much of its effect by "neutralizing" LPS by binding tightly to the LPS molecule. Further, 3-azalipid $\mathrm{X}$, the diamino analog of lipid $\mathrm{X}$, displays inhibitory effects at low concentrations identical to lipid X but LPS-like (agonist) activity at higher concentrations. The demonstration of both agonist and antagonist effect by the same molecule, chemically related to LPS, suggests the possibility that a receptor-ligand interaction may be important in producing neutrophil priming.

Lipid X, in increasing concentrations, shifts the LPS dose response curve of human neutrophils rightward, necessitating higher concentrations of LPS to achieve the same rate of $\mathrm{O}_{2}^{-}$ release. This pattern of response to a blocking agent is consistent with competitive inhibition, though a role for effects on membrane fluidity and permeability have not been completely ruled out. In addition, the respiratory burst represents a complex cascade in which multiple binding sites with more than one type of interaction could be present. The possibility also remains that lipid X or a metabolite of lipid X may be effecting a step in cell activation removed from the major site of LPS action, although it does not appear to influence $\mathrm{O}_{2}^{-}$production in the absence of LPS.

These experiments provide evidence that LPS-induced neutrophil priming may be caused by the specific binding of LPS to cell component(s) or receptor(s) involved in the regulation of the respiratory burst, and that structurally similar molecules may be able to compete with LPS for these putative site(s) resulting in a unique anti-LPS effect. Regardless of the exact mechanism(s) of this action, lipid $X$ is representative of a group of compounds with novel properties that may be useful in the treatment of gram-negative sepsis and septic shock. Further, lipid $\mathrm{X}$ and its analogs can be utilized to probe the molecular basis of the cellular actions of LPS and provide a more complete understanding of the pathogenesis of the septic shock syndrome.

\section{Acknowledgments}

The authors are grateful to Dr. Joel Moss and Dr. Henry Masur for their critical review of this manuscript. We wish to thank Dr. David Alling for his much appreciated guidance with statistical analysis and Ms. Sue Oremland and Kathy Kiefer for their excellent secretarial assistance.

\section{References}

1. Wolff, S. M., and J. V. Bennett. 1974. Gram-negative rod bacteremia. N. Engl. J. Med. 291:733.

2. Scheckler, W. E. 1978. Nosocomial infections in a community hospital. Arch. Intern. Med. 138:1792-1794.

3. Kreger, B. E., D. E. Craven, P. C. Carling, and W. R. McCabe. 1980. Gram-negative bacteremia III. Reassessment of etiology, epidemiology and ecology in 612 patients. Am. J. Med. 68:332-343. 
4. Bryan, C. S., K. L. Reynolds, and E. R. Brenner. 1983. Analysis of 1,186 episodes of gram-negative bacteremia in nonuniversity hospitals: the effects of antimicrobial therapy. Rev. Infect. Dis. 5:629-638.

5. McCabe, W. R., and R. N. Olans. 1981. Shock III. Gram-negative bacteremia: predisposing factors, pathophysiology and treatment. In Current Clinical Topics in Infectious Diseases. J. S. Remington and M. N. Swartz, editors. McGraw-Hill Book Co., New York. 2:121-150.

6. McCabe, W. R. 1984. Antibiotics and endotoxic shock. Bull. N.Y. Acad. Med. 51:1084-1095.

7. Ryan, J. L. 1985. Microbial factors in pathogenesis: lipopolysaccharide. In Contemporary Issues in Infectious Diseases. M. A. Sande and R. K. Root, editors. Churchill Livingstone, Inc. New York. 4:13-25.

8. Schumer, W. 1976. Steroids in the treatment of clinical septic shock. Ann. Surg. 184:333-341.

9. Sprung, C. L., P. V. Cavalis, E. H. Marcial, M. Pierce, M. A. Gelbard, W. M. Long, R. C. Duncan, M. D. Tendler, and M. Karpf. 1984. The effects of high dose corticosteroids in patients with septic shock: prospective, controlled study. N. Engl. J. Med. 311:1137-1143.

10. Holaday, J. W., and A. I. Faden. 1978. Naloxone reversal of endotoxin hypotension suggests role of endorphins in shock. Nature (Lond.). 275:450-451.

11. Peters, W. P., M. W. Johnson, P. A. Friedman, and W. E. Mitch. 1981. Pressor effects of naloxone in septic shock. Lancet. i:529-532.

12. Fletcher, J. R., and P. W. Ramwell. 1977. Modification by aspirin and indomethacin of the hemodynamic and prostaglandin releasing effects of E. coli endotoxin in the dog. Br. J. Pharmacol. 61:175-181.

13. Halushka, P. V., W. C. Wise, and J. A. Cook. 1983. Studies on the beneficial effects of aspirin in endotoxic shock. Relationship to inhibition of arachidonic acid metabolism. Am. J. Med. 74:91-96.

14. Corrigan, J. J., and J. F. Kiernat. 1979. Effect of polymyxin B sulfate on endotoxin activity in a gram-negative septicemia model. Pediatr. Res. 13:48-51.

15. Ziegler, E. J., J. A. McCutchan, J. Fierer, M. P. Glauser, J. C. Sadoff, H. Douglas, and A. I. Braude. 1982. Treatment of gram-negative bacteremia and shock with human antiserum to a mutant Escherichia coli. N. Engl. J. Med. 307:1225-1230.

16. Baumgartner, D. J., M. P. Glauser, J. A. McCutchan, E. J. Ziegler, G. V. Melle, M. R. Klauber, M. Vogt, E. Muehlen, R. Luethy, R. Chiolero, and S. Geroulanos. 1985. Prevention of gram-negative shock and death in surgical patients by antibody to endotoxin core glycolipid. Lancet. ii:59-63.

17. Takayama, K., N. Qureshi, P. Mascagni, L. Anderson, and C. R. H. Raetz. 1983. Glucosamine-derived phospholipids in Escherichia coli. J. Biol. Chem. 258:14245-14252.

18. Raetz, C. R. H. 1984. The enzymatic synthesis of lipid A: molecular structure and biologic function of monosaccharide precursors. Rev. Infect. Dis. 6:463-471.

19. Nishijima, M., and C. R. H. Raetz. 1981. Characterization of two membrane-associated glycolipids from a Escherichia coli deficient in phosphatidylglycerol. J. Biol. Chem. 256:10690-10696.

20. Kiso, M., H. Ishida, and A. Hasegawa. 1984. Synthesis of biologically active, novel monosaccharide analogs of lipid A. Agric. Biol. Chem. 48:251-252.

21. Kusumoto, S., M. Yamamoto, and T. Shiba. 1984. Chemical synthesis of lipid $\mathrm{X}$ and Lipid $\mathrm{Y}$, acylglucosamine 1-phosphates isolated from Escherichia coli mutants. Tetrahedron Lett. 25:3727-3730.

22. Takayama, K., N. Qureshi, P. Mascagni, M. A. Nashed, L. Anderson, and C. R. H. Raetz. 1983. Fatty acyl derivatives of glucosamine-1-phosphate in Escherichia coli and their relation to lipid A. $J$. Biol. Chem. 258:7379-7385.

23. Ray, B. L., G. Painter, and C. R. H. Raetz. 1984. The biosynthesis of gram-negative endotoxin: formation of lipid A disaccharides from monosaccharide precursors in extracts of Escherichia coli. $J$. Biol. Chem. 259:4852-4859.

24. Nishijima, M., I. Amano, J. Akamatsu, K. Akagawa, T. To- kuraga, and C. R. H. Raetz. 1984. Macrophage activation by monosaccharide precursors of Escherichia coli lipid A. Proc. Natl. Acad. Sci. USA. 82:282-286.

25. Wightman, P. D., and C. R. H. Raetz. 1984. The activation of protein kinase $\mathrm{C}$ by biological active lipid moieties of lipopolysaccharide. J. Biol. Chem. 259:10048-10052.

26. Raetz, C. R. H., S. Purcell, and K. Takayama. 1983. Molecular requirements for B lymphocyte activation by Escherichia coli lipopolysaccharide. Proc. Natl. Acad. Sci. USA. 80:4624-4628.

27. Golenbock, D. T., J. A. Will, C. R. H. Raetz, and R. A. Proctor. 1985. Pharmacokinetic data of lipid $X$ in adult sheep. In Twenty-fifth Interscience Conference on Antimicrobial Agents and Chemotherapy. 25:215. (Abstr.)

28. Golenbock, D. T., J. E. Leggett, C. R. H. Raetz, and R. A. Proctor. 1986. Lipid X protects mice from death due to endotoxin and gram-negative septicemia. Clin. Res. 34:518A. (Abstr.)

29. Proctor, R. A., J. A. Will, K. E. Burhop, and C. R. H. Raetz. 1986. Protection of mice against lethal endotoxemia by a lipid A precursor. Infect. Immun. 52:905-907.

30. Guthrie, L. A., L. C. McPhail, P. M. Henson, and R. B. Johnston. 1984. Priming of neutrophils for enhanced release of oxygen metabolites by bacterial lipopolysaccharides. J. Exp. Med. 160:16561671.

31. Haslett, C., L. A. Guthrie, M. M. Kopaniak, R. B. Johnston, Jr., and P. M. Henson. 1985. Modulation of multiple neutrophil functions by preparative methods or trace concentrations of bacterial lipopolysaccharide. Am. J. Pathol. 119:101-110.

32. Atkins, E., and C. Heijn, Jr. 1965. Studies on tuberculin fever III: mechanisms involved in the release of endogenous pyrogen in vitro. J. Exp. Med. 122:207-235.

33. Babior, B. M., R. S. Kipnes, and J. T. Curnutte. 1973. Biological defense mechanisms: the production by leukocytes of superoxide, a potent bactericidal agent. J. Clin. Invest. 52:741-744.

34. Johnston, R. B., Jr., B. B. Keele, Jr., H. P. Misra, J. E. Lehmeyer, R. L. Baehner, and K. V. Rajagopalan. 1975. The role of superoxide anion generation in phagocytic bactericidal activity: studies with normal and chronic granulomatous disease leukocytes. J. Clin. Invest. 55:1357-1372.

35. Massey, V. 1959. The microestimation of succinate and the extinction coefficient of cytochrome c. Biochim. Biophys. Acta. 34:255-256.

36. Mann, H. B. 1945. Nonparametric tests against trend. Econometrica. 13:245-259.

37. Nelson, L. S. 1977. Tables for testing ordered alternatives in an analysis of variance. Biometrika. 64:333-338.

38. Morrison, D. C., and D. M. Jacobs. 1976. Binding of polymyxin B to the lipid A portion of bacterial LPS. Immunochemistry. 13:813-818.

39. Morrison, D. C., and J. A. Rudbach. 1981. Endotoxin-cell membrane interactions leading to transmembrane signals. Contemp. Top. Mol. Immunol. 8:187-218.

40. Wilson, M. E. 1985. Effects of bacterial endotoxin on neutrophil function. Rev. Infect. Dis. 7:404-418.

41. Levin, J., T. E. Poore, N. P. Zauber, and R. S. Oser. 1970. Detection of endotoxin in the blood of patients with sepsis due to gram-negative bacteria. N. Engl. J. Med. 283:1313-1316.

42. Danner, R. L., R. J. Elin, J. M. Hosseini, T. Schlesinger, P. Roach, and J. E. Parrillo. 1986. Endotoxemia is associated with many clinical manifestations of septic shock in humans. Clin. Res. 34:410A. (Abstr.)

43. Bentler, B., N. Krochin, I. W. Milsark, C. Luedke, and A. Cerami. 1986. Control of cachetin (tumor necrosis factor) synthesis: mechanism of endotoxin resistance. Science (Wash. DC). 232:977980.

44. Duff, G. W., and E. Atkins. 1982. The detection of endotoxin by in vitro production of endogenous pyrogen: comparison with limulus amebocyte lysate gelation. J. Immunol. Methods. 52:323-331.

45. Beutler, B., I. W. Milsark, and A. C. Cerami. 1985. Passive 
immunization against cachectin/tumor necrosis factor protects mice from lethal effect of endotoxin. Science (Wash. DC). 229:869-871.

46. Dinarello, C. A. 1985. An update on human interleukin-1: from molecular biology to clinical relevance. J. Clin. Immunol. 5:287-297.

47. Parrillo, J. E. 1986. Septic shock in humans: recent insights regarding pathogenesis, cardiovascular dysfunction, and therapy. Crit. Care Med. 7:383-435.

48. Smedly, L. A., M. G. Tonnesen, R. A. Sandhaus, C. Haslett, L. A. Guthrie, R. B. Johnston, Jr., P. M. Henson, and G. S. Worthen. 1986. Neutrophil-mediated injury to endothelial cells: enhancement by endotoxin and essential role of neutrophil elastase. J. Clin. Invest. 77:1233-1243.

49. Carr, C., and D. C. Morrison. 1984. Lipopolysaccharide interaction with rabbit erythrocyte membranes. Infect. Immun. 43:600606.

50. Carr, C., and D. C. Morrison. 1985. Mechanism of polymyxin
B mediated lysis of lipopolysaccharide treated erythrocytes. Infect. Immun. 49:84-89.

51. Morrison, D. C., Z. G. Oades, and D. DiPietro. 1981. Endotoxin-initiated membrane changes in rabbit platelets. Prog. Clin. Biol. Res. 62:47-64.

52. Munford, R. S., and C. L. Hall. 1986. Detoxification of bacterial lipopolysaccharides (endotoxins) by a human neutrophil enzyme. Science (Wash. DC). 234:203-205.

53. Aderem, A. A., M. M. Keum, E. Pure, and Z. A. Cohn. 1986. Bacterial lipopolysaccharides, phorbol myristate acetate, and zymosan induce the myristoylation of specific macrophage proteins. Proc. Natl. Acad. Sci. USA. 83:5817-5821.

54. Nishizuka, Y. 1983. Phospholipid degredation and signal translation for protein phosphorylation. Trends Biochem. Sci. 8:13-16.

55. Nishizuka, Y. 1984. The role of protein kinase $C$ in cell surface signal transduction and tumour promotion. Nature (Lond.). 308:693698. 